\title{
Quantitative Mobility Assessment for Fall Risk Prediction in Dementia: A Systematic Review
}

\author{
Elham Dolatabadi ${ }^{a}$ Karen Van Ooteghem ${ }^{a}$ Babak Taati ${ }^{a-c}$ \\ Andrea laboni ${ }^{a} d$ \\ aToronto Rehabilitation Institute - University Health Network, Toronto, ON, Canada; \\ ${ }^{b}$ Department of Computer Science, University of Toronto, Toronto, ON, Canada; \\ 'Institute of Biomaterials and Biomedical Engineering, Toronto, ON, Canada; \\ ${ }^{\mathrm{d} D e p a r t m e n t}$ of Psychiatry, University of Toronto, Toronto, ON, Canada
}

\section{Keywords}

Fall · Gait · Balance · Dementia · Quantitative assessment

\begin{abstract}
Background: Impairments of gait and balance often progress through the course of dementia, and are associated with increased risk of falls. Summary: This systematic review provides a critical analysis of the evidence linking quantitative measures of gait and balance to fall risk in older adults with dementia. Various instrumented measures of gait and postural stability including gait speed and non-instrumented performance measures including Timed Up and Go were shown to be capable of distinguishing fallers from non-fallers. Key Messages: Existing reviews indicate that impairments of gait and balance are associated with increased risk of falls in cognitively intact older people. There are inconsistencies, however, regarding the characteristics most predictive of a fall. In order to advance fall prevention efforts, there is an important need to understand the relationship between gait, balance, and fall risk, particularly in high-risk populations such as individuals with dementia.

(c) 2018 S. Karger AG, Basel
\end{abstract}


Dolatabadi et al.: Quantitative Mobility Assessment for Fall Risk Prediction in Dementia

\section{Introduction}

Falls have a wide-ranging impact on functional ability, caregiver burden, and quality of life, and they are a leading cause of injury, loss of independence, and mortality in people with dementia. The mortality rate in the year after hip fracture for an individual with dementia is nearly $30 \%$ [1]. Understanding what puts a person at risk of falling is of great importance and can help reduce falls and fall-related injuries in this population $[1,2]$. Falls prevention efforts however, rely on adequate assessment of falls risk including the use of clinically meaningful metrics and protocols appropriate for individuals with dementia.

Beyond extrinsic risk factors for falls, such as footwear, medication use, and environmental hazards [3-5], individuals with dementia are at increased risk of falls related to intrinsic changes in sensory, neuromuscular, and cognitive functions. Walking is a task that requires cognitive input, particularly in the domains of attention and executive functioning [6-9]. Balance, the ability to maintain postural control, also requires cognitive input [10]. Hence, changes in cognitive function contribute to gait disturbances, balance disorders, and increased fall risk $[2,11]$. Mobility limitations such as unsteady gait and balance impairment are more common in people with dementia compared with cognitively healthy older adults, and are also associated with an increased risk of falls $[3,10,12,13]$. Individuals with dementia have been shown to have slower gait speed, shorter stride length, greater cycle-to-cycle gait variability, larger body sway, and higher center of pressure (COP) variability $[6,8,11,12$, 14-20]. Accordingly, information about gait and balance characteristics could be informative in predicting the risk of falling [10].

Quantitative measures of gait or postural stability can be captured using a variety of instruments or sensors, and have been shown to be associated with falls risk in communitydwelling older adults without dementia [21, 22]. However, there have been some inconsistencies between studies in terms of the characteristics that are predictive of a fall. For example, much of this work has found that gait variability is able to discriminate between fallers and non-fallers [10,12, 16, 23, 24]. Conversely, Brach et al. [25] concluded that the coefficient of variability in step time and stance time do not differentiate these groups. Moreover, Camicioli and Licis [26] found that only cadence and not any other measure of gait was predictive of falls. Similarly, in a systematic review conducted by Menant et al. [7], it was reported that single and dual task gait analysis have equal predictive capacity, and therefore, there is no need to include dual task tests in fall risk assessments. This finding is in contrast to the results of a recent review by Muir-Hunter et al. [27] which concluded that the association between gait performance and fall risk was stronger under dual- versus single-task conditions. In addition to gait variability, gait speed [28] and movement path variability (tortuosity) [29] have been confirmed to help with fall prediction in cognitively healthy older adults. Maki [16] also showed that a combination of postural sway and gait measures significantly improved the prediction of future falls. There are several possibilities that could account for the inconsistencies reported in the literature including the heterogeneity of the populations studied (community-dwelling to nursing home residents) and varied approaches to administration of the functional assessments and the analysis of outcome measures.

In addition to these inconsistencies, existing studies are limited by the exclusion of participants with dementia; thus, findings are not generalizable to this population. Deficits in memory, attention, comprehension, praxis, and motor skills have an important impact on performance of functional assessments $[11,30]$. Many of the assessment tools or instruments used in studies to measure gait and balance as predictors of falls may not be appropriate for individuals with dementia, who have an impaired ability to follow complex directions and variability in their motivation and attention to the task [12]. Consequently, there is a pressing 


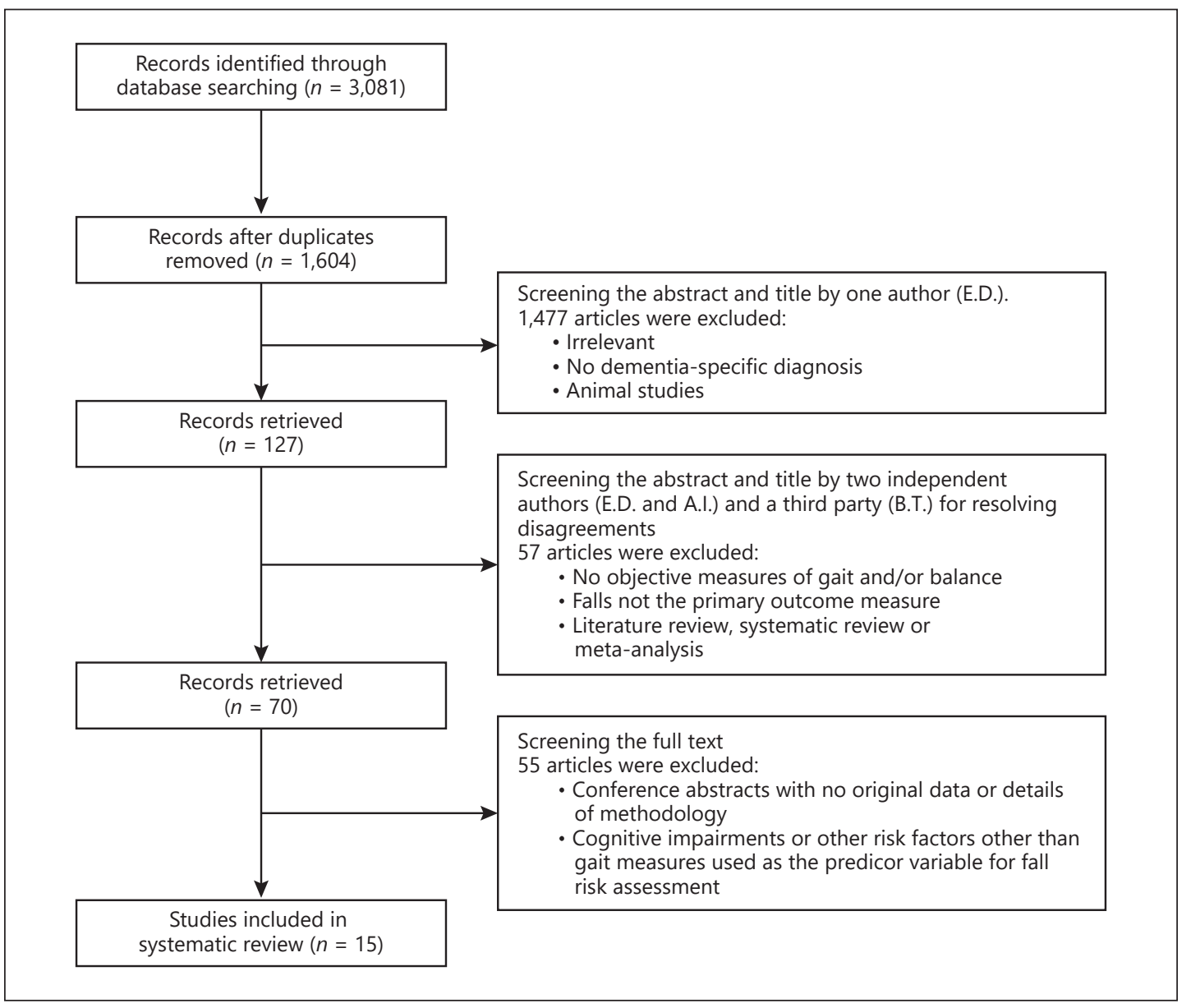

Fig. 1. Study selection flow diagram.

need for valid and reliable gait and balance measures to help identify individuals with dementia at highest risk for falls.

The primary aim of this study was thus to complete a systematic review of quantitative measures of gait and balance related to the prediction of falls, with a focus on older adults with dementia. It was our objective to identify those quantitative measures of gait and balance that were both most strongly associated with falls risk and feasible to measure in this population.

\section{Methods}

Search Strategy

The literature search was performed in July 2017. The following electronic databases were searched for studies in English: CENTRAL, CINAHL, Compendex, Embase, Medline, PsychINFO, and Web of Science. MeSH subject terms and keywords were used to capture the concepts of "Dementia" and "Falls" and "Gait/Balance" (see Appendix 1 for a full list of search terms). English-language prospective and retrospective clinical studies were selected if they: (1) included older adults with a diagnosis of dementia (mean age 60 years and older), (2) measured gait or balance, and (3) examined falls as an outcome. Studies solely focused on mild cognitive impairment were excluded. 
Study Selection and Analysis

Search results were combined and checked for duplicates using the EndNote package (Endnote v. 8.0.2; Thomson Reuters, CA, USA). After duplicates were removed, the articles' titles and abstracts were reviewed by one of the authors (E.D.) for initial screening and irrelevant studies excluded. Next, two authors (E.D. and A.I.) independently reviewed article titles and abstracts, and potentially relevant articles were included for full text review. All disagreements were resolved by a third party (B.T.). A flow diagram of the study selection is shown in Figure 1. All authors of the paper (E.D., K.V., B.T., and A.I.) contributed to the analysis of selected articles.

\section{Results}

Sixty-one articles were selected for full text review. A total of 15 studies met the inclusion criteria. Table 1 summarizes participant characteristics and settings for the studies included in this review. As shown in Table 1, sample size varied widely among articles, from 20 to 358 participants, with 2 studies [31, 32] including data from agematched control participants.

\section{Study Settings}

Seven studies were conducted in long-term care facilities [12, 13, 23, 26, 29, 33, 34], and 6 studies were community-based [2, 24, 31, 32, 35, 36]. Two studies [37, 38] included mixed settings with a majority of participants residing in the community and the remainder living in long-term care.

\section{Study Design}

Study design varied across articles (see Table 2): 3 studies were cross-sectional with one baseline assessment [31, 32, 38], 9 studies involved a gait/balance assessment at baseline (also cross-sectional) with a follow-up for up to 2, 3, 4, 5, 12, or 24 months to record occurrence of fall incidents [2, 12, 13, 24, 26, 32, 34-37], and 2 studies included repeated gait and balance data collection throughout the follow-up period [23,39]. One study [29] collected gait and balance measures at baseline and captured a measure of tortuosity continuously over a 1-year period. Two studies included data from healthy control participants (no dementia) or participants with mild dementia in their analysis $[32,38]$.

\section{Quantitative Measures of Gait and Balance}

The type of quantitative gait and balance measures used to assess fall risk fell into two categories: (1) 7 studies included instrumented quantitative measures of gait and balance (e.g., accelerometers) [2, 23, 24, 26, 31, 36, 38] and (2) 5 studies included non-instrumented, performance measures (e.g., 6-min walk) [13, 32, 34, 35, 37]. A combination of instrumented and performance measures of gait and balance was used in 3 studies [12, 29, 39] (Table 2). Instrumented measures were captured using an electronic walkway (5 studies), force plates (2 studies), wearable sensors (accelerometers and gyroscope) (3 studies), and a motion capture system (1 study). Overall, the quantitative measures obtained from these instruments were spatiotemporal gait ( 7 studies), postural sway (4 studies), and a measure of path tortuosity (1 study). Functional mobility measures included as examples, Timed Up and Go (TUG), timed 180 and turn, Berg Balance Score (BBS), Physical Performance Test (PPT), 6-min walk, tandem gait (10 studies), and dynamic/static balance measures such as Tinetti Performance Oriented Mobility Assessment (POMA), mCTSIB, Romberg test, functional reach, grip strength, and 4-step balance (7 studies). 


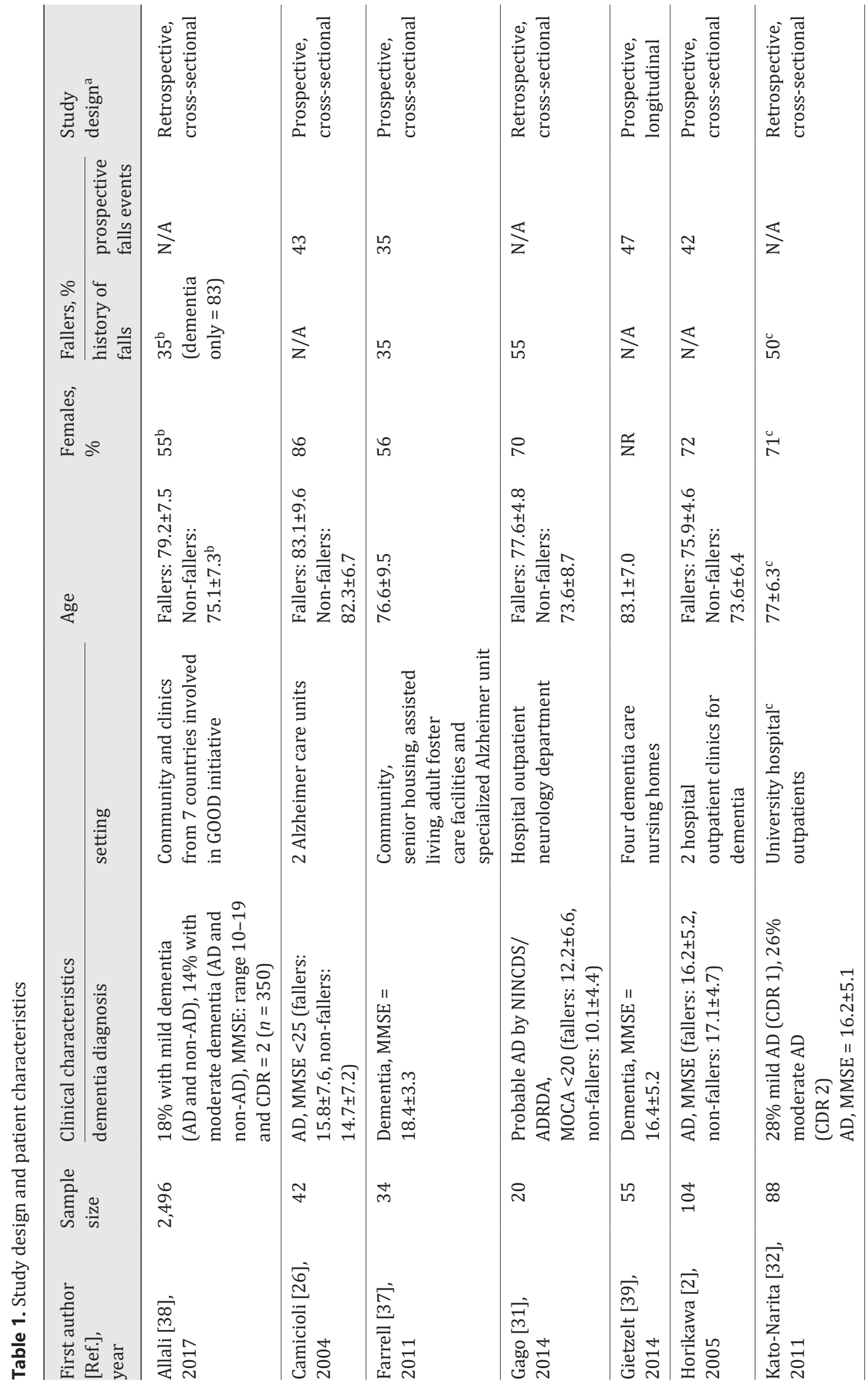


Dementia

and Geriatric
Cognitive Disorders
Dement Geriatr Cogn Disord 2018;45:353-367

DOI: 10.1159/000490850

2018 S. Karger

Dolatabadi et al.: Quantitative Mobility Assessment for Fall Risk Prediction in Dementia

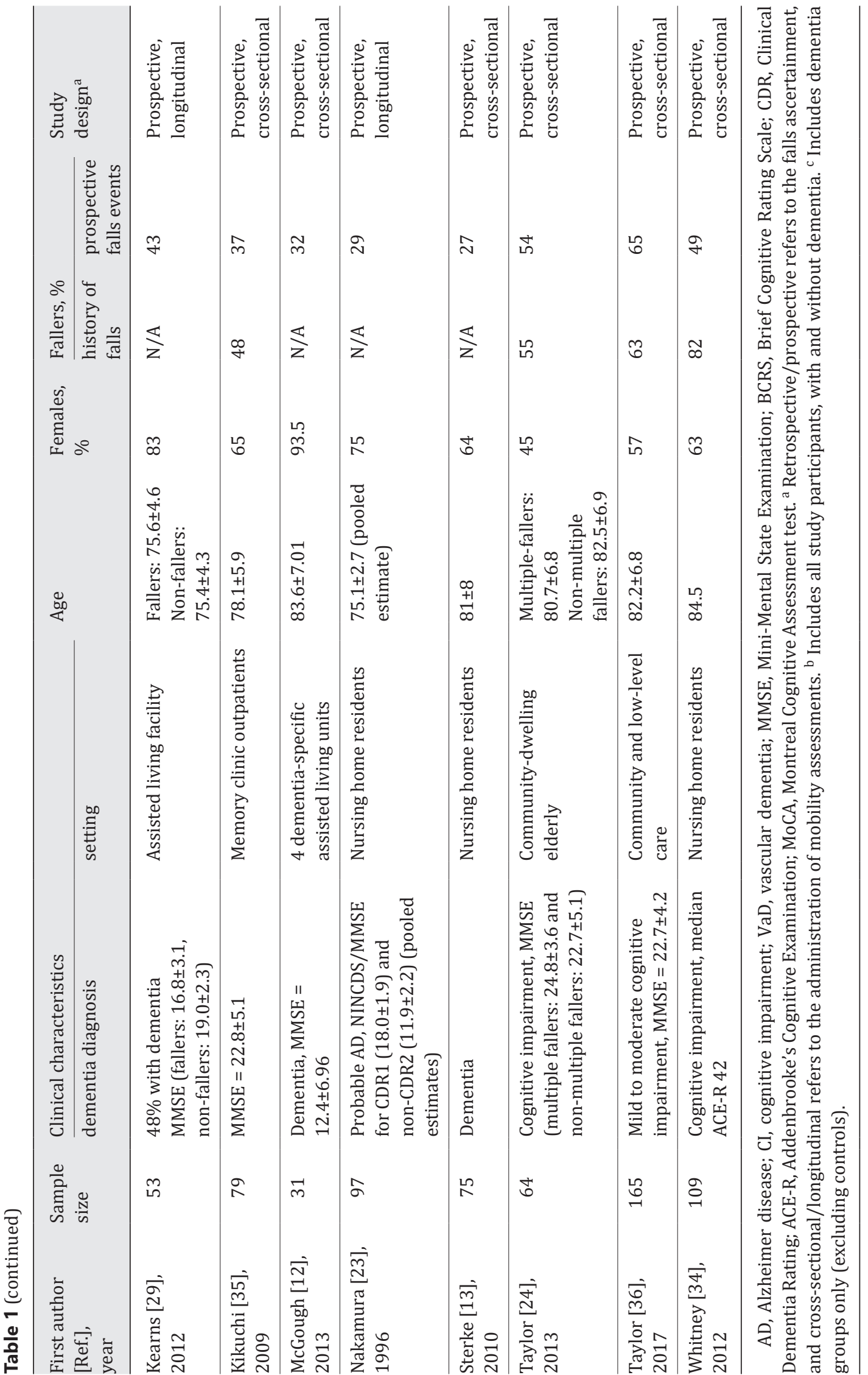


Dementia

and Geriatric
Cognitive Disorders
Dement Geriatr Cogn Disord 2018;45:353-367

DOI: 10.1159/000490850

(c) 2018 S. Karger AG, Base

www.karger.com/dem

Dolatabadi et al.: Quantitative Mobility Assessment for Fall Risk Prediction in Dementia

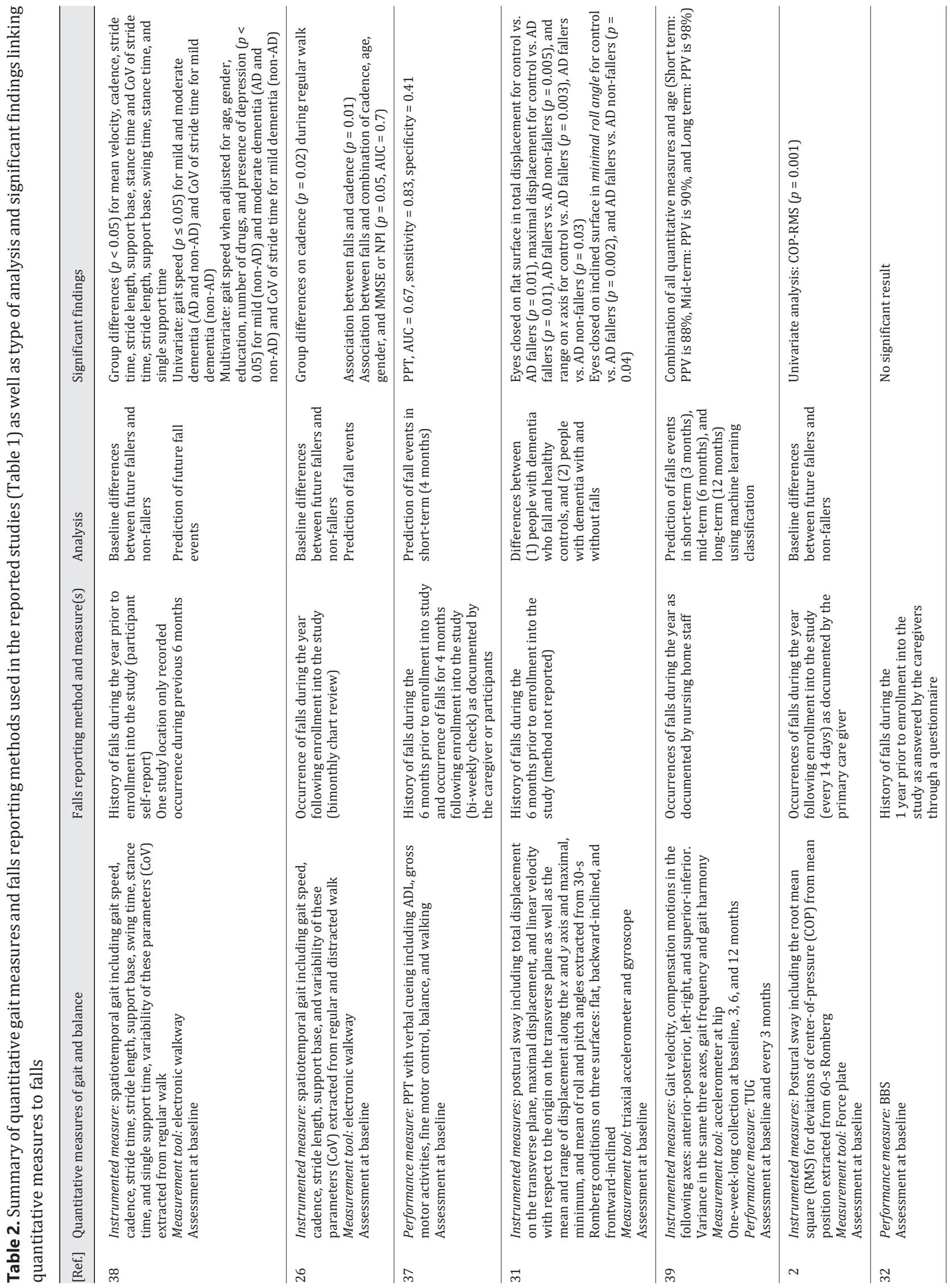


Dementia

Cognitive Disorders
Dement Geriatr Cogn Disord 2018;45:353-367

360

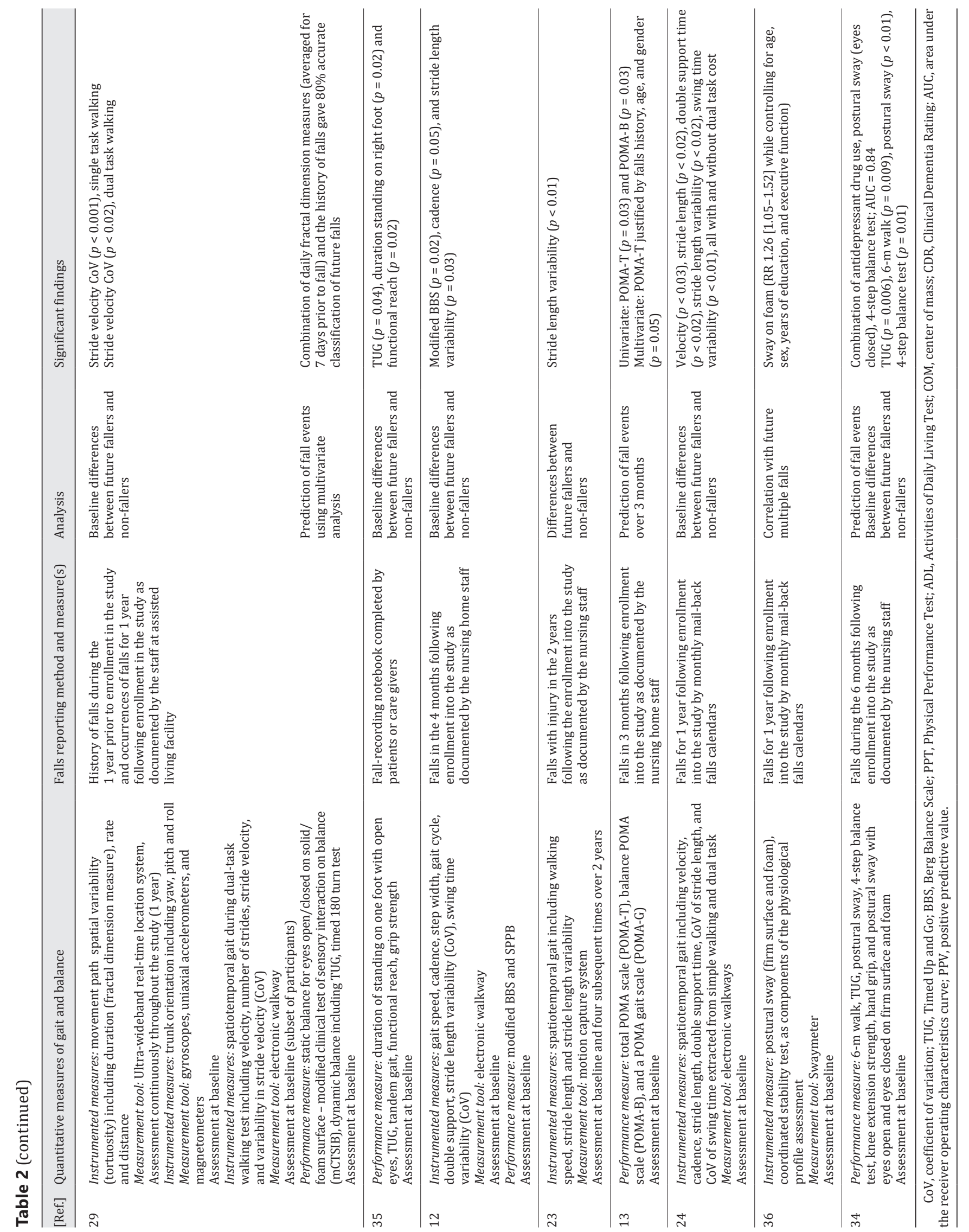




\section{Fall Risk Analysis}

Various types of analyses such as univariate (14 studies), multivariate (7 studies), correlational ( 2 studies), and predictive modelling ( 7 studies) were used for linking quantitative measures to falls. In total, 3 studies were solely based on the retrospective history of falls (prior to baseline performance measurement) $[31,32,38]$ and the remaining 12 studies used prospective falls data. Five studies used both retrospective and prospective fall information for their analysis $[2,12,13,23,24,26,29,34-37,39]$.

Among the spatiotemporal measures of gait used in the studies of this review, two measures - gait speed (cadence or velocity) $[12,24,26,29,38,39]$ and stride length (average or variability) $[12,23,24,29,38]$ - were often found to differentiate between fallers and non-fallers. These two measures were utilized either as single variables $[12,23,24,26,29$, $38]$ or multiple variables combined with age and/or gender [26, 29, 38, 39] based on both retrospective history and prospective occurrences of falls. Among balance measures, postural sway such as COP displacement, sway on foam, and Romberg test [2, 31, 34, 36] were also significantly associated with both retrospective history and prospective occurrences of falls.

Mixed results were found between studies in terms of the predictive ability of TUG for fall prediction. While 2 studies $[34,35]$ recommended TUG as a successful predictor of future falls, 2 other studies $[29,39]$ failed to find that the TUG predicts falls in the dementia population. In a recent study conducted by Kearns et al. [29], movement path tortuosity was found to be a significant predictor of a future fall when combined with previous fall history. For short-term (less than 6 months) prediction of falls, gait speed, COP displacement and acceleration, PPT and POMA were significantly associated with falls [13, 37, 39].

\section{Discussion}

The aim of this systematic review was to determine whether or not quantitative measures of gait and balance can be used as predictors of falls among older adults with dementia. Our findings align with outcomes of previous reviews examining fall prediction in healthy older adults $[28,40]$, and provide important additions to the literature by (1) focusing on dementia populations, (2) examining both instrumented and non-instrumented measures of gait and balance, and (3) focusing on falls as the outcome measure. Our findings support the growing body of evidence regarding the effectiveness of mobility assessment in identifying individuals with dementia at high risk for falls [12, 26, 29, 31, 32, 34, 36, 38]. Gait speed (either cadence or velocity), stride length (mainly variability measures), and postural sway (total displacement of COP) were employed commonly in successful fall prediction and/or retrospective modelling. Kearns et al. [29] showed that changes in path tortuosity in the 7 days before a fall may be a useful addition to the existing measures of gait and balance. Path tortuosity is an indicator whether an older adult with dementia is capable of walking in a relatively straight line and was shown to be a more accurate predictor of falls compared to gait speed, while performing slightly worse than stride time variability in the subset of participants for whom these data were available.

Performance-based measures including TUG, BBS, Romberg test, and POMA were used less frequently than instrumented gait and balance measures and were not as successful as instrumented tools in retrospectively and prospectively distinguishing fallers from nonfallers. Six studies $[13,26,34,36,38,39]$ in this review combined measures of gait and balance (either instrumented or performance based) with other known risk factors such as falls history, age, and gender in order to improve the accuracy of fall predictions. We also know that other risk factors, such as use of psychotropic medication, sleep disturbance, and behav- 
ioral symptoms, also contribute to falls in older adults with dementia [4, 5, 41, 42]. Despite the fact that these three risk factors are prevalent in this population and might influence the outcomes of interest, the studies in this review either did not include these factors or did not find them to be significant.

Several significant limitations were identified related to clinical feasibility and generalizability of the studies in our review. Many of the studies were cross-sectional or involved a very short follow-up after a single assessment. The heterogeneity of the disease, and its progressive, deteriorating course, challenges the ability of any single cross-sectional assessment to identify those most at risk of falling. The majority of studies in this review included a one-time mobility assessment (at the baseline), and only 2 studies $[23,39]$ repeated gait and balance parameters at different time points throughout the study. The absence of ongoing monitoring critically limits measures' responsiveness to changes over time, as the predictive value of quantitative assessment of gait declines with time from assessment [43]. There was only one study in which a quantitative gait measure (path tortuosity) was captured continuously throughout the study [29].

Methodological issues relating to the reporting of falls are also common in these studies. Seven studies [2, 24, 32, 35-38] in this review followed up any falls incident through selfreporting or notebooks which were filled out by either participants or their primary caregiver over the period of the study. However, self-reporting of falls history in a geriatric population, especially among individuals with cognitive impairment, has problems with reliability [33].

Another limitation of the studies included in this review concerns the approach to statistical analysis for identifying predictors of falls. The analysis of instrumented measures of gait and balance is challenging due to the highly dimensional, temporally dependent, highly variable, and non-linear nature of human movement patterns [44]. The human movement episodes exhibit inter-trial, temporal dependency as well as intra-trial correlation. Moreover, for cross-sectional analysis, there is high variability between subjects. As such, linear and other commonly used statistical models such as logistic regression, ANOVA, or $\chi^{2}$ test cannot extract useful information from episodes of human motion data and are unlikely to result in accurate prediction of falls [45]. Machine learning algorithms, including dynamic temporal models such as recurrent neural network or hidden Markov models, may be better tools to detect gait and balance anomalies in individuals' walking patterns that are predictive of impending falls [46].

From the perspective of feasibility as a clinical tool for falls risk assessment, we found that many of the studies that included instrumented assessment made use of technologies in laboratory settings (such as motion capture systems) or controlled environments (such as on flat, instrumented walkways) for capturing gait and balance data. These types of assessments are rarely clinically available, and have the limitation of being somewhat artificial in nature; therefore, they may not reflect the cognitive and physical demands of naturally moving around the environment. In the study by Kearns et al. [29], 36\% of older adult participants refused to walk on the instrumented walkway to complete a gait and balance assessment. However, the tortuosity measures which were obtained by wearable sensors during participants' daily activities were available for all participants. Two other studies in the review also used wearable sensors to measure gait [31,39]. Of the studies that used wearable technology, 2 of them $[29,39]$ reported the need to follow-up with the participants regarding use of the sensors (i.e., adherence, appropriate mounting/orientation, etc.) and failures in their data collection. Others in the literature have reported that older adults transferred the wearable sensors between various body locations due to discomfort [46, 47]. Moreover, at present, the algorithms used to process data from wearable sensors can have difficulty identifying gait episodes that are asymmetric or irregular, which limits their utility in the advanced dementia population. 
Regarding non-instrumented performance measures which were reported in this review, there are concerns about their reliability and validity in people with dementia, particularly individuals at the advanced stage of disease [10,27]. Most of the studies in this review recruited community-dwelling individuals with mild to moderate dementia (MMSE in the range of 12-20). There were very few studies that included individuals with more advanced dementia, or focused on those who lived in long-term care facilities, which is a population at particular risk of serious, recurrent, and injurious falls [23, 32, 38]. As many of the assessments reported in this review require some understanding, cooperation, and motivation on the part of the participant, the results may not be generalizable to those at more advanced stages of the disease. Although BBS, TUG, Functional Reach Test, 6-min walk test, and PPT are used in people with dementia, their responsiveness to change in performance in longitudinal assessments has not been established. There is some disagreement among researchers and clinicians on the acceptability of performance measures for a dementia population [27, 48-50]. Five studies $[2,13,26,34,37]$ identified some difficulties in the administration of the assessment due to the cognitive impairment of the participants. One study [12] employed the modified version of the performance assessment tool in order to accommodate for the attention and memory deficit in individuals with dementia. Taken together, it is likely that the performance tests discussed in this review would require some minor modifications or be inappropriate for use depending on the type and severity of the dementia [27].

\section{Opportunities for Future Research}

In this review, we have demonstrated that quantitative assessments of mobility can contribute to an assessment of falls risk in people with dementia. However, there is a need to develop an approach to assessment that is reliable in this population and that is sensitive to changes in physical status. One way to do this would be to develop a technology that can monitor changes in quantitative measures of gait and balance repeatedly over time. Such a system in the home environment could monitor naturalistic movements remotely, and detect changes in response to interventions or therapies. Examples of emerging technologies include wearables such as accelerometers $[29,51]$, vision-based data capture $[52,53]$, or real-time location sensor networks [29]. Vision-based technologies blend into older adult living environments and track their movement without the need for wearables. There is also the opportunity to use technology for falls monitoring [54,55], to avoid the shortcomings that accompany retrospective recall and self- or informal-reporting, and provide quantitative data about fall mechanics. By using technology for the collection of large sets of quantitative mobility data, there is also the opportunity for non-linear analysis of gait and balance performance for dynamic fall risk assessment. Recently, machine learning algorithms [5659] have been investigated as effective solutions to describe the underlying pattern within datasets and how they are related to gait analysis and falls risk assessment. While technology has the potential to improve the assessment of mobility in dementia, issues such as obtrusiveness, participant compliance, and equipment and software cost have the potential to limit its clinical usefulness.

\section{Conclusion}

Impaired gait, postural instability, and falls are important safety issues for older adults with dementia. Our systematic review provides evidence that quantitative measures of gait and postural stability can contribute to falls risk prediction in this population. There is a need for clinically feasible, responsive, assessment approaches and technologies to help further develop predictive models in dementia. 


\section{Acknowledgement}

The authors would like to acknowledge Jessica Babineau, MLIS information specialist from Toronto Rehabilitation Institute - University Health Network, for her assistance with the development and execution of the literature search. The authors also thank Twinkle Arora for proofreading the manuscript.

\section{Disclosure Statement}

The authors have no conflicts or financial or non-financial interest.

\section{Funding Sources}

This study was partially funded by the Alzheimer's Association, Brain Canada, and AGE-WELL Canada's technology and aging network.

\section{Author Contributions}

Screening the articles: Elham Dolatabadi, Babak Taati, and Andrea Iaboni. Analysis and interpretation of article: Elham Dolatabadi, Karen Van Ooteghem, and Andrea Iaboni. Drafting the article: Elham Dolatabadi. Revising it critically: Elham Dolatabadi, Karen Van Ooteghem, Babak Taati, and Andrea Iaboni. Providing final approval of the version to be published: Elham Dolatabadi, Karen Van Ooteghem, Babak Taati, and Andrea Iaboni.

\section{Appendix 1}

The search strategies consisted of a mix of subject headings (such as $\mathrm{MeSH}$ ) and keywords relating to the concepts of "Dementia" and "Falls" and "Gait/Balance" as shown below:

1 exp Dementia/

2 Delirium, Dementia, Amnestic, Cognitive Disorders/

3 Neurocognitive Disorders/

4 Cognition Disorders/

5 dement*.tw,kw.

6 alzheimer*.tw,kw.

7 ((cognitive or cognition or neurocognitive) adj2 (disorder* or impair* or deficit* or defect*)).tw,kw.

8 (lewy* adj2 bod*).tw,kw.

9 ('organic brain disease' or 'organic brain syndrome').tw,kw.

10 (pick* adj2 disease).tw,kw.

11 (creutzfeldt or jcd or cjd).tw,kw.

12 Huntington Disease/

13 huntington*.tw,kw.

14 binswanger*.tw,kw.

15 Auditory Perceptual Disorders/

16 ((auditory or acoustic or psychoacoustical) adj2 disorder*).tw,kw.

17 (senile or senility).tw,kw.

18 or $/ 1-17$

19 Accidental Falls/

20 (fall or falls or faller or fallen or fallers or falling or fell).tw,kw.

21 (slip or slips or slipped or slipping).tw,kw.

22 (trip or trips or tripped or tripping).tw,kw.

23 (stumble or stumbles or stumbled or stumbling).tw,kw.

24 (tumble or tumbles or tumbled or tumbling).tw,kw.

25 ((lose or loses or loss) adj5 footing).tw,kw.

26 or $/ 19-25$ 


\section{References}

1 Seitz DP, Gill SS, Gruneir A, et al: Effects of dementia on postoperative outcomes of older adults with hip fractures: a population-based study. J Am Med Dir Assoc 2014;15:334-341.

2 Horikawa E, Matsui T, Arai H, Seki T, Iwasaki K, Sasaki H: Risk of falls in Alzheimer's disease: a prospective study. Intern Med 2005;44:717-721.

3 Van Doorn C, Gruber-Baldini AL, Zimmerman S, et al: Dementia as a risk factor for falls and fall injuries among nursing home residents. J Am Geriatr Soc 2003;51:1213-1218.

4 Sylliaas H, Selbæk G, Bergland A: Do behavioral disturbances predict falls among nursing home residents? Aging Clin Exp Res 2012;24:251-256.

5 St George RJ, Delbaere K, Williams P, Lord SR: Sleep quality and falls in older people living in self-and assistedcare villages. Gerontology 2009;55:162-168.

6 Sheridan PL, Hausdorff JM: The role of higher-level cognitive function in gait: executive dysfunction contributes to fall risk in Alzheimer's disease. Dement Geriatr Cogn Disord 2007;24:125-137.

7 Menant JC, Schoene D, Sarofim M, Lord SR: Single and dual task tests of gait speed are equivalent in the prediction of falls in older people: a systematic review and meta-analysis. Ageing Res Rev 2014;16:83-104.

8 Allali G, Assal F, Kressig RW, Dubost V, Herrmann FR, Beauchet O: Impact of impaired executive function on gait stability. Dement Geriatr Cogn Disord 2008;26:364-369.

9 Kearney FC, Harwood RH, Gladman JR, Lincoln N, Masud T: The relationship between executive function and falls and gait abnormalities in older adults: a systematic review. Dement Geriatr Cogn Disord 2013;36:20-35.

10 Morgan D, Funk M, Crossley M, Basran J, Kirk A, Dal Bello-Haas V: The potential of gait analysis to contribute to differential diagnosis of early stage dementia: current research and future directions. Can J Aging 2007;26: 19-32.

11 Pettersson A, Olsson E, Wahlund L-O: Motor function in subjects with mild cognitive impairment and early Alzheimer's disease. Dement Geriatr Cogn Disord 2005;19:299-304.

12 McGough EL, Logsdon RG, Kelly VE, Teri L: Functional mobility limitations and falls in assisted living residents with dementia: physical performance assessment and quantitative gait analysis. J Geriatr Phys Ther 2013;36: 78-86.

13 Sterke CS, Huisman SL, van Beeck EF, Looman CW, van der Cammen TJ: Is the Tinetti Performance Oriented Mobility Assessment (POMA) a feasible and valid predictor of short-term fall risk in nursing home residents with dementia? Int Psychogeriatr 2010;22:254-263.

14 Wittwer JE, Webster KE, Menz HB: A longitudinal study of measures of walking in people with Alzheimer's Disease. Gait Posture 2010;32:113-117.

15 Sheridan PL, Solomont J, Kowall N, Hausdorff JM: Influence of executive function on locomotor function: divided attention increases gait variability in Alzheimer's disease. J Am Geriatr Soc 2003;51:1633-1637.

16 Maki BE: Gait changes in older adults: predictors of falls or indicators of fear? J Am Geriatr Soc 1997;45:313320.

17 Merlo A, Zemp D, Zanda E, et al: Postural stability and history of falls in cognitively able older adults: The Canton Ticino study. Gait Posture 2012;36:662-666. 
18 Suttanon P, Hill KD, Said CM, LoGiudice D, Lautenschlager NT, Dodd KJ: Balance and mobility dysfunction and falls risk in older people with mild to moderate Alzheimer disease. Am J Phys Med Rehabil 2012;91:12-23.

19 Manckoundia P, Pfitzenmeyer P, d'Athis P, Dubost V, Mourey F: Impact of cognitive task on the posture of elderly subjects with Alzheimer's disease compared to healthy elderly subjects. Mov Disord 2006;21:236241.

20 Bramell-Risberg E, Jarnlo G-B, Minthon L, Elmståhl S: Lower gait speed in older women with dementia compared with controls. Dement Geriatr Cogn Disord 2005;20:298-305.

21 Nevitt MC, Cummings SR, Kidd S, Black D: Risk factors for recurrent nonsyncopal falls: a prospective study. JAMA 1989;261:2663-2668.

22 Berg WP, Alessio HM, Mills EM, Tong C: Circumstances and consequences of falls in independent communitydwelling older adults. Age Ageing 1997;26:261-268.

23 Nakamura T, Meguro K, Sasaki H: Relationship between falls and stride length variability in senile dementia of the Alzheimer type. Gerontology 1996;42:108-113.

24 Taylor ME, Delbaere K, Mikolaizak AS, Lord SR, Close JC: Gait parameter risk factors for falls under simple and dual task conditions in cognitively impaired older people. Gait Posture 2013;37:126-130.

25 Brach JS, Berlin JE, VanSwearingen JM, Newman AB, Studenski SA: Too much or too little step width variability is associated with a fall history in older persons who walk at or near normal gait speed. J Neuroeng Rehabil 2005;2:21.

26 Camicioli R, Licis L: Motor impairment predicts falls in specialized Alzheimer care units. Alzheimer Dis Assoc Disord 2004;18:214-218.

27 Muir-Hunter SW, Graham L, Montero Odasso M: Reliability of the Berg Balance Scale as a clinical measure of balance in community-dwelling older adults with mild to moderate Alzheimer disease: a pilot study. Physiother Can 2015;67:255-262.

28 Deandrea S, Bravi F, Turati F, Lucenteforte E, La Vecchia C, Negri E: Risk factors for falls in older people in nursing homes and hospitals. A systematic review and meta-analysis. Arch Gerontol Geriatr 2013;56:407415.

29 Kearns WD, Fozard JL, Becker M, et al: Path tortuosity in everyday movements of elderly persons increases fall prediction beyond knowledge of fall history, medication use, and standardized gait and balance assessments. J Am Med Dir Assoc 2012;13:665.e7-665.e13.

30 Blankevoort CG, Van Heuvelen MJ, Boersma F, Luning H, De Jong J, Scherder EJ: Review of effects of physical activity on strength, balance, mobility and ADL performance in elderly subjects with dementia. Dement Geriatr Cogn Disord 2010;30:392-402.

31 Gago MF, Fernandes V, Ferreira J, et al: Postural stability analysis with inertial measurement units in Alzheimer's disease. Dement Geriatr Cogn Disord Extra 2014;4:22-30.

32 Kato-Narita EM, Nitrini R, Radanovic M: Assessment of balance in mild and moderate stages of Alzheimer's disease: implications on falls and functional capacity. Arq Neuropsiquiatr 2011;69:202-207.

33 McMichael KA, Vander Bilt J, Lavery L, Rodriguez E, Ganguli M: Simple balance and mobility tests can assess falls risk when cognition is impaired. Geriatr Nurs 2008;29:311-323.

34 Whitney J, Close JC, Jackson SH, Lord SR: Understanding risk of falls in people with cognitive impairment living in residential care. J Am Med Dir Assoc 2012;13:535-540.

35 Kikuchi R, Kozaki K, Iwata A, Hasegawa H, Toba K: Evaluation of risk of falls in patients at a memory impairment outpatient clinic. Geriatr Gerontol Int 2009;9:298-303.

36 Taylor ME, Lord SR, Delbaere K, Kurrle SE, Mikolaizak AS, Close JCT: Reaction time and postural sway modify the effect of executive function on risk of falls in older people with mild to moderate cognitive impairment. Am J GeriatrPsychiatry 2017;25:397-406.

37 Farrell MK, Rutt RA, Lusardi MM, Williams AK: Are scores on the physical performance test useful in determination of risk of future falls in individuals with dementia? J Geriatr Phys Ther 2011;34:57-63.

38 Allali G, Launay CP, Blumen HM, et al: Falls, cognitive impairment, and gait performance: results from the GOOD Initiative. J Am Med Dir Assoc 2017;18:335-340.

39 Gietzelt M, Wolf KH, Marschollek M, Haux R: Predicting falls in people with dementia using accelerometry - a one-year prospective multi-center field study. Biomed Tech/Biomed Eng 2014;59:S712-S715.

40 Kröpelin TF, Neyens JC, Halfens RJ, Kempen GI, Hamers JP: Fall determinants in older long-term care residents with dementia: a systematic review. Int Psychogeriatr 2013;25:549-563.

41 Iaboni A, Flint AJ: The complex interplay of depression and falls in older adults: a clinical review. Am J Geriatr Psychiatry 2013;21:484-492.

42 Flint AJ, Iaboni A, Mulsant BH, et al: Effect of sertraline on risk of falling in older adults with psychotic depression on olanzapine: results of a randomized placebo-controlled trial. Am J Geriatr Psychiatry 2014;22: 332-336.

43 Klenk J, Becker C, Palumbo P, et al: Conceptualizing a dynamic fall risk model including intrinsic risks and exposures. J Am Med Dir Assoc 2017;18:921-927.

44 Chau T: A review of analytical techniques for gait data. Part 1: fuzzy, statistical and fractal methods. Gait Posture 2001;13:49-66.

45 Iluz T, Weiss A, Gazit E, et al: Can a body-fixed sensor reduce Heisenberg's uncertainty when it comes to the evaluation of mobility? Effects of aging and fall risk on transitions in daily living. J Gerontol A Biomed Sci Med Sci 2015;71:1459-1465. 
46 Rajagopalan R, Litvan I, Jung T-P: Fall prediction and prevention systems: recent trends, challenges, and future research directions. Sensors 2017;17:2509.

47 Mathie MJ, Coster AC, Lovell NH, Celler BG, Lord SR, Tiedemann A: A pilot study of long-term monitoring of human movements in the home using accelerometry. J Telemed Telecare 2004;10:144-151.

48 Blankevoort CG, Van Heuvelen MJ, Scherder EJ: Reliability of six physical performance tests in older people with dementia. Phys Ther 2013;93:69-78.

49 Ries JD, Echternach JL, Nof L, Gagnon Blodgett M: Test-retest reliability and minimal detectable change scores for the timed "up and go" test, the six-minute walk test, and gait speed in people with Alzheimer disease. Phys Ther 2009;89:569-579.

50 Van Ooteghem K, Musselman K, Gold D, Marcil MN, Keren R, Tartaglia MC, Flint AJ, Iaboni A: Evaluating Mobility in Advanced Dementia: A Scoping Review and Feasibility Analysis. Gerontologist July 5, 2018, Epub ahead of print.

51 Caby B, Kieffer S, de Saint Hubert M, Cremer G, Macq B: Feature extraction and selection for objective gait analysis and fall risk assessment by accelerometry. Biomed Eng Online 2011;10:1.

52 Parra-Dominguez GS, Taati B, Mihailidis A: 3D human motion analysis to detect abnormal events on stairs. Second International Conference on 3D Imaging, Modeling, Processing, Visualization and Transmission (3DIMPVT), Zurich, October 2012.

53 Dolatabadi E, Taati B, Mihailidis A: Automated classification of pathological gait after stroke using ubiquitous sensing technology. 38th Annual International Conference of the IEEE Engineering in Medicine and Biology Society (EMBC), Orlando, August 2016.

54 Belshaw M, Taati B, Snoek J, Mihailidis A: Towards a single sensor passive solution for automated fall detection. 33rd Annual International Conference of the IEEE Engineering in Medicine and Biology Society, Boston, 2011.

55 Stone EE, Skubic M: Fall detection in homes of older adults using the Microsoft Kinect. IEEE J Biomed Health Informatics 2015;19:290-301.

56 Dolatabadi E, Mansfield A, Patterson K, Taati B, Mihailidis A: Mixture-model clustering of pathological gait patterns. IEEE J Biomed Health Informatics 2017;21:1297-1305.

57 Lai DT, Levinger P, Begg RK, Gilleard WL, Palaniswami M: Automatic recognition of gait patterns exhibiting patellofemoral pain syndrome using a support vector machine approach. IEEE Trans Inf Technol Biomed 2009;13:810-817.

58 Silva J, Madureira J, Tonelo C, et al: Comparing machine learning approaches for fall risk assessment. BIOSIGNALS 2017, Porto, February 2017.

59 Marschollek M, Rehwald A, Wolf K, et al: Sensor-based fall risk assessment - an expert "to go." Methods Inf Med 2011;50:420-426. 